\title{
An algorithm based on ViBe for detecting moving objects in industrial field
}

\author{
LUO Rui-guang ${ }^{1, a}$, YANG Jiang-hua ${ }^{1, b}$ \\ ${ }^{1}$ DaLian University of Technology, LiaoNing, DaLian, China \\ aluoruiguang@126.com, b524750766@qq.com
}

Keywords: Computer Vision; Motion Detection; Industrial Field; ViBe; Gost; Interference

\begin{abstract}
In many industrial fields, there always exists mechanical regular movements, for example, the movements of reciprocating pumps in an oil field, which brings limitation for the detection. The tranditional methods including background subtraction cannot be used directly. In order to fit the scene with regular reciprocating movements, a new method based on ViBe is proposed in this paper. The new method divides the frame into two parts, dynamic region and static region. Improved algorithm of $\mathrm{ViBe}$ is used to detect moving target in static region. The improved algorithm has a strong anti-interference performance and effectly eliminate the ghost area, which makes the improved algorithm fit different industral scene.
\end{abstract}

\section{Introduction}

With the continuous development of artificial intelligence and automatic control in recent years, computer vision has more and more applications. In computer vision, moving target detection and tracking is particularly important, which are used in military, transportation, intelligent monitoring and other fields. It is a topic at the forefront of technology with a variety of aspects [1]. Detection of moving targets is also facing some problems at the same time such as the elimination of ghost, adapting the changing background environment, and the shaking of camera. These problems bring some limitations to accurate detection.

For the detection of moving targets containing reciprocating background, such as reciprocation pumps in CBM field [2], shown in Fig. 1. This paper divides the background frame into static and dynamic regions, and improved ViBe algorithm is used to detect moving targets in static section, which saves computing resources of the system[3], improves the system instantaneity and accuracy of detection.

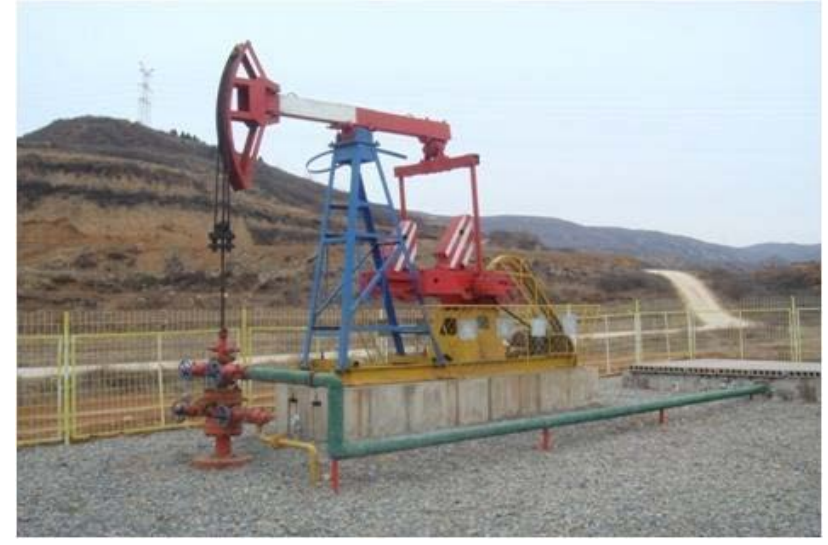

Fig. 1 Pump with reciprocating movements

\section{Review of the algorithm of ViBe}

In 2009 Olivier and Make Fan proposed ViBe (Visual Background Extractor) algorithm[4]. It was the first time for cluster theory to be applied in target detection. ViBe includes the following several respects. 


\section{Pixel model of ViBe}

ViBe background model requires only one frame in a video to be established. In the model denoted by $M(x)$, each pixel x will have a set of samples, the number of samples is $n$. Background model of each pixel is defined as shown in Eq. 1:

$$
M(x)=\left\{v_{1}, v_{2}, \ldots v_{\mathrm{n}-1}, v_{n}\right\}
$$

In the literature proposed in 2009, the number of samples $n$ is 20. And 20 samples are randomly selected from 8 neighbors of pixel $\mathrm{x}$ as a background model, shown in Fig. 2.

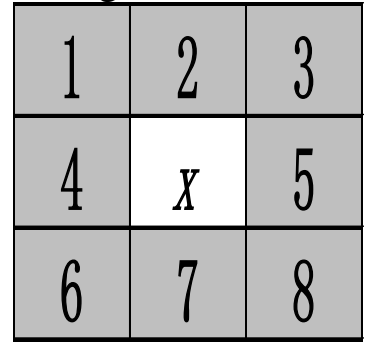

Fig. 2 Eight neighbor pixels of $\mathrm{x}$

\section{Classification process}

Detection of foreground pixels is a process of classification, and it needs a criterion for a pixel to classify whether it is part of the foreground or the background. ViBe imposes the influence of a value in the polychromatic space to be limited to the local neighborhood.

We denote by $v(x)$ the value at time t of the pixel $\mathrm{x}$. To classify a value $v(x)$, we compare it to its closest values among the set of samples by defining a sphere $\mathrm{S}_{\mathrm{R}}(v(x))$ of radius $\mathrm{R}$ centered on $v(x)$, shown in Fig. 3.

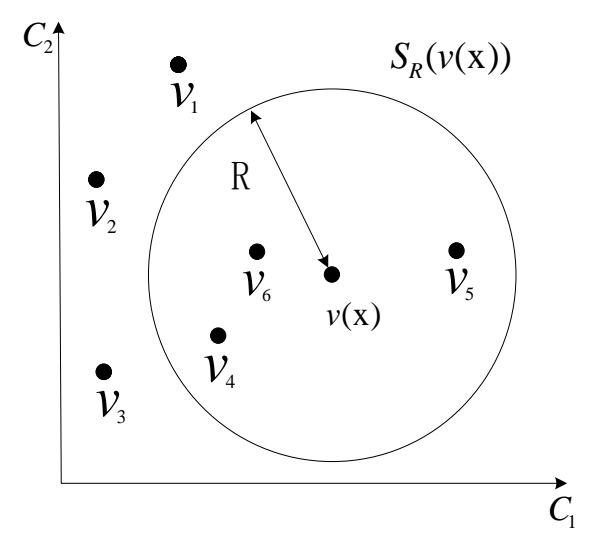

Fig. 3 Sphere area centered on $v(x)$

A pixel value is then classified as background if the cardinality, denoted \#, of the set intersection of this sphere and the set of samples is above a given threshold Min, and in binary image this pixel will be set 0, or it will be set 1 if classified as foreground, as shown in Eq. 2.

\section{Model update}

$$
\begin{cases}\mathrm{x}=0, & \#\left[\mathrm{~S}_{\mathrm{R}}(v(x)) \cap M(x)\right] \geq \operatorname{Min} ; \\ \mathrm{x}=1, \quad \#\left[\mathrm{~S}_{\mathrm{R}}(v(x)) \cap M(x)\right]<\operatorname{Min} ;\end{cases}
$$

ViBe uses random update policy to replace pixels in the model with background pixel values. This ensures that the likelihood of presence of each sample value in the model monotonically decreases, but also to ensure an accurate estimate with fewer sample values.

\section{Improved ViBe}

In many industrial fields, there always exist mechanical regular movements, for example, the movements of reciprocating pumps in an oil field, which brings limitation for the detection. The tranditional methods including background subtraction cannot be used directly. In order to fit the scene with regular movements, a new method based on ViBe is proposed. 


\section{Pixel model with static and dynamic regions}

Before establishing ViBe background model, dynamic region can be set aside by the algorithm of frame difference ${ }^{[5]}$. Interval between each frame is decided by the speed of reciprocation motion. After the detection of several frames, dynamic region can be marked completely and then motion detection can be applied in static region.

\section{Expanded pixel model}

In ViBe algorithm, 20 samples is randomly selected from 8 neighbors of pixel x, which means at least one neigborhood is chosen more than twice. Excessive concentrated samples will increase the probability of an erroneous judgement ${ }^{[6]}$, especially in outdoor industry environment and make the algorithm sensitive to tiny motion. For example the puny motion of grass and cloud or the tiny change of sunlight will produce much noise interference[7].

Improved ViBe expands the range of sample selection, so that a number of small changes of pixel position can be absorbed in outdoor scene. At the same time, pixel drift is random, so the expansion region should fit in a circular area. As shown in Fig. 4, the expansion region is 20 neighbors of pixel.

\begin{tabular}{|c|c|c|c|c|}
\hline & 1 & 2 & 3 & \\
\hline 4 & 5 & 6 & 7 & 8 \\
\hline 9 & 10 & $x$ & 11 & 12 \\
\hline 13 & 14 & 15 & 16 & 17 \\
\hline & 18 & 19 & 20 & \\
\hline
\end{tabular}

\section{Hierarchical classification}

Fig. 4 Twenty neighbor pixels fitting the sphere

The expanded pixel model requires more samples to ensure each element has a prosibility to be chosen. In outdoor industral environment, expanded pixel model can absorb some nosie interference, but also increse the probability of erroneous judgement. To solve this problem, this paper uses hierarchical classification.

Improved ViBe no longer uses a single boundary in Fig. 3. In the coordinate system, the closer the current pixel and the midpoint of background model, the contribution of this point is greater. This paper sets the boundary $\frac{1}{2} R$, shown in Fig. 5 .

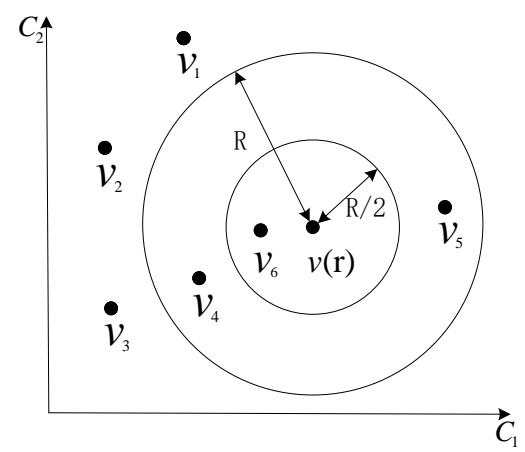

Fig. 5 Area segmentation with a boundary of $\frac{1}{2} R$

After the hierarchical classification, this paper donates by $a, b$ different contribution in each section, defined as shown in Eq. 3.

$$
\left\{\begin{array}{c}
\text { Min }+a, \quad\left|\mathrm{~S}_{\mathrm{R}}(v(x))-M(x)\right| \leq \frac{1}{2} R \\
\operatorname{Min}+b, \quad \frac{1}{2} R<\left|\mathrm{S}_{\mathrm{R}}(v(x))-M(x)\right| \leq R
\end{array}\right.
$$

The parameter $a, b(a>b)$ can be adjusted according to the actual different situations, for example in indoor scene, we can increase $a$, and reduce $b$, to enhance the accuracy, in outdoor scene, we can reduce $a$ and increse $b(a>b)$ to enhance the anti-interference performance. 


\section{Elimination of ghost}

In ViBe algorithm, if an object in original background moves away, original position of the object will produce a moving object that does not correspond to any of the detection area ${ }^{[8]}$, called a "ghost", as shown in Fig. 6.

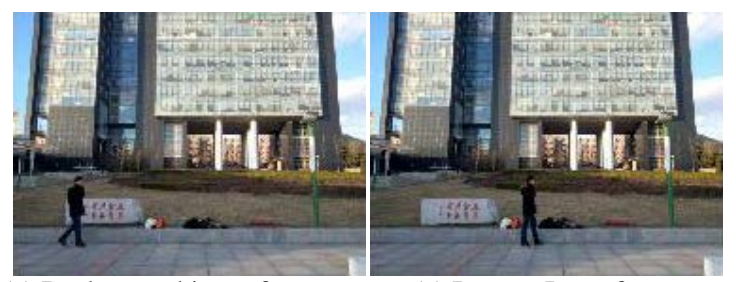

(a) Background input frame

(c) Present Input frame

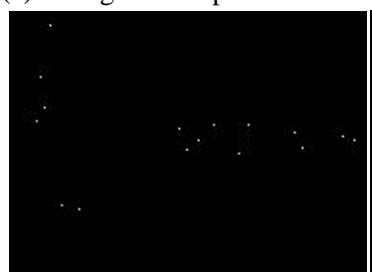

(b) background output frame

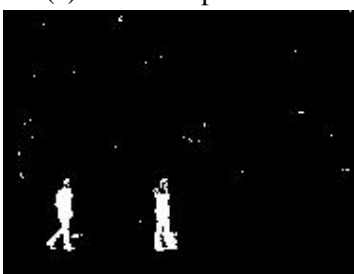

(d)Present output frame

Fig. 6 Gost created by ViBe algorithm

To eliminate ghost, this paper proposes a new strategy. When the moving target is detected, but the position has not changed and this situation keeps more than $k$ consecutive frames, then this target will be judged as a ghost, and be updated by corresponding region in present frame. This strategy can eliminate ghost effectively and won't impact the following detection when other targets move through ghost area.

\section{Experimental results}

The advantage of an algorithm can be judged from accuracy, robustness and real-time aspects[9]. In order to verify the effects of improved algorithm, this article will compare the rusults with unmodified ViBe algorithm and kernel density estimation(KDE) in different scenarios.

Experiments were performed with two groups of video frames. The first group of video frames are used to confirm the ablity of suppression of noise interference and elimination of ghost, as shown in Fig. 7.

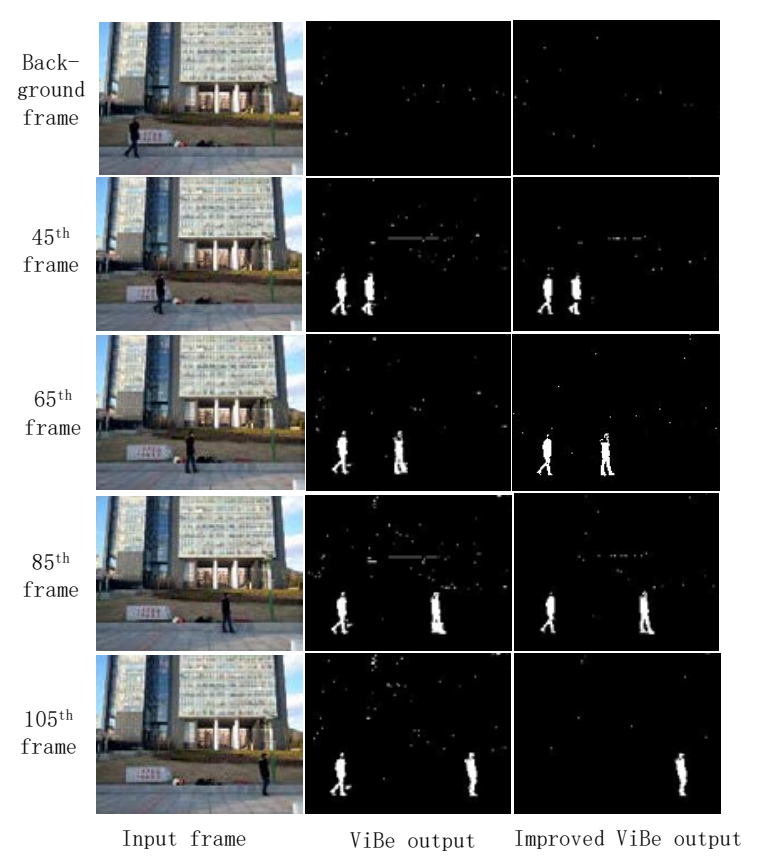

Fig. 7 The performance of anti-interference and gost eliminating of improved ViBe

As can be seen from the results of the simulation, improved ViBe algorithm can better suppress interference caused by slight movements of the camera and the noise caused by tiny motion of 
background. Meanwhile, the ghost is eliminated completely in 105th frame after three frames.

The second group frames are from industrial field with reciprocating motion. Improved ViBe uses 20 frames to mark the dynamic region and then detects moving target in static region. Results are as shown in Fig. 8.
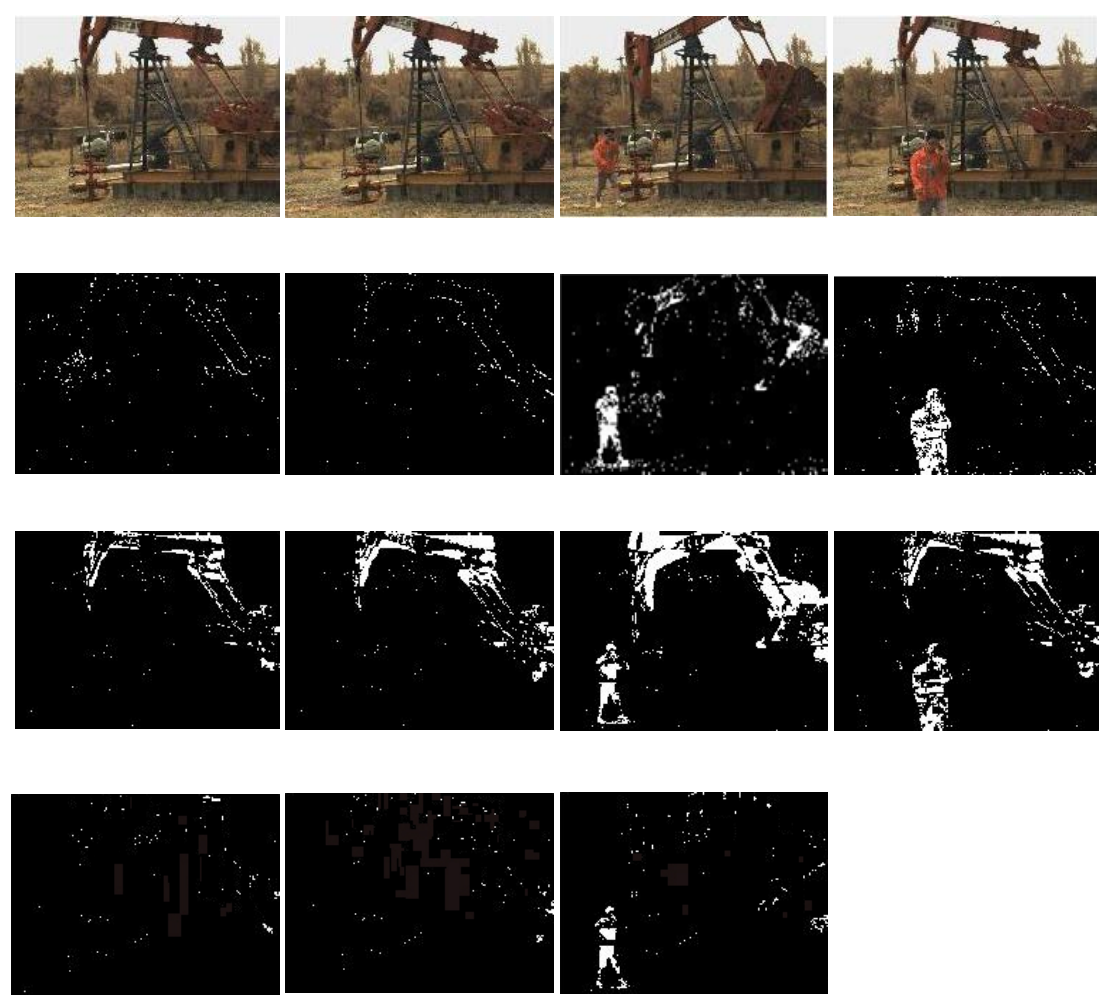

Fig. 8 Output of improved ViBe comparison with ViBe and KDE

The result shows improved ViBe has a good performance of marking dynamic region and can detect moving target accurately. What's more, improved ViBe uses less time than KDE, almost half of the time of KDE, when processing each frame.

\section{Conclusions}

This paper proposes an improved ViBe algorithm to fit outdoor scene containing regular reciprocating movements, including the following three aspects:

(1) Establish a pixel model with static and dynamic regions and remove the objects with regular reciprocating movements.

(2) Expand the pixel model reasonably and propose a hierarchical classification to make the model fit in different scenes.

(3) Introduce a counter to compulsively update the model to eliminate ghost.

After the improvement shown in the above, the simulation results reveal that improved ViBe has a good performance of fitting in the outdoor scene with regular reciprocating motion.

\section{Acknowledgements}

This paper is financially supported by Major National Science and Technology Programs of China in the "Twelfth Five-Year" Plan period (2011ZX05039).

\section{References}

[1] ZHANG Ling-ying Research of moving target based on computer vision, Technology 
Innovation and Application[J], 2013(34):15-15.

[2] CUI Lu, Significance and Principle Analysis of CBM. Science and Technology Innovation Herald[J], 2009(22): 120

[3] Olivier Barnich, Marc Van Droogenbroeck. A Universal Background Subtraction Algorithm for Video Sequence, IEEE.Transation on Image Processing, 2011, Vol.20 NO.6. Page(s):1709 1724

[4] Olivier Barnich, Marc Van Droogenbroeck. ViBE: A powerful random technique to estimate the background in video sequences. IEEE.2009 Page(s): 945-948.

[5] LI Gang. New moving target detection method based on background differencing and coterminous frames differencing. Chinese Journal of Scientific Instrument[J], 2006, 27(8):961-964

[6] YU Ye. Evibe: An improved Vibe algorithm for detecting moving objects. Chinese Journal of Scientific Instrument[J], VoL35, No.4, Apr.014.

[7] M Van Droogenbroeck, O Paquot. Background Subtraction: Experiments and Improvements for ViBe. IEEE.2012. Pages: 32-37.

[8] HU Xiao-ran, SUN Han. Novel Moving Object Detection Method Based on ViBe. Computer Science [J]. 2014,41 (2):149-152.

[9] WAN Ying, HAN Yi, LU Han-qing. The Methods for Moving Object Detection. Computer Simulation[J], 2006, 23(10):221-226. 